\title{
Transient response in high-resolution Brillouin-based distributed sensing using probe pulses shorter than the acoustic relaxation time
}

\author{
V. Lecoeuche, D. J. Webb, C. N. Pannell, and D. A. Jackson \\ Applied Optics Group, School of Physical Sciences, University of Kent, Canterbury CT2 7NR, UK
}

Received October 18, 1999

\begin{abstract}
We perform numerical simulations on a model describing a Brillouin-based temperature and strain sensor, testing its response when it is probed with relatively short pulses. Experimental results were recently published [e.g., Opt. Lett. 24, 510 (1999)] that showed a broadening of the Brillouin loss curve when the probe pulse duration is reduced, followed by a sudden and rather surprising reduction of the linewidth when the pulse duration gets shorter than the acoustic relaxation time. Our study reveals the processes responsible for this behavior. We give a clear physical insight into the problem, allowing us to define the best experimental conditions required for one to take the advantage of this effect. () 2000 Optical Society of America

OCIS codes: $\quad 060.2370,190.5890,290.5900$.
\end{abstract}

Stimulated Brillouin scattering couples two counterpropagating optical waves, the pump and the Stokes waves, and an acoustic wave. Interaction is maximum for a precise value of the frequency shift between the two optical waves, which itself depends on the temperature and strain conditions in the material. Distributed sensing in an optical fiber is commonly obtained by use of a cw pump and a Stokes-shifted probe pulse. One measures the intensity of the transmitted pump field as a function of the frequency shift to resolve the Brillouin gain spectrum. Positional information is obtained through a time-domain analysis; shorter probe pulses increase the spatial resolution. Unfortunately, but expectedly, the Brillouin gain spectral width broadens as the pulse duration is reduced. ${ }^{1,2}$ This broadening leads to difficulties in the determination of the resonant Brillouin shift and therefore to a larger error in the measurement for submeter spatial resolution. However, it was found recently that reducing the pulse duration to values smaller than the acoustic relaxation time entails a sudden decrease of the spectral width., ${ }^{3,4}$ In those studies it was suggested that a spatial resolution of $10 \mathrm{~cm}$ was achievable, with a relatively good strain or temperature accuracy.

In this Letter we present the results of numerical simulations highlighting the mechanisms responsible for the observed spectral width variation. These theoretical findings show good agreement with the experimental results described in Ref. 3. The present study also reveals the crucial influence of a totally unsuspected parameter, that is, the extinction ratio of the electro-optic modulator (EOM) used to generate the pulses. We show that a modest extinction ratio (20$30 \mathrm{~dB}$ ) results in spectral narrowing with ultrashort pulses, as reported in Ref. 3, whereas for a high (infinite) extinction ratio the gain linewidth broadens, as expected from a standard Fourier analysis of the system. ${ }^{1}$

The theoretical model used to simulate the interactions in the sensing fiber is the following ${ }^{4,5}$ :

$$
\begin{aligned}
\partial E_{p} / \partial_{t}+\partial E_{p} / \partial z & =-E_{s} E_{a}, \\
\partial E_{s} / \partial_{t}-\partial E_{s} / \partial z & =E_{p} E_{a}{ }^{*}, \\
\partial E_{a} / \partial_{t}+[1+i \Delta(z)] E_{a} & =E_{p} E_{s}{ }^{*},
\end{aligned}
$$

where $E_{p}, E_{s}$, and $E_{a}$ are the normalized pump, Stokes, and acoustic fields, respectively. The fiber attenuation, which is not relevant for lengths in the meter range, is neglected. The acoustic-wave convection term is neglected, since the acoustic velocity is negligible compared with the optical velocity. $\Delta(z)$ is the detuning parameter normalized to the acoustic damping rate, $\gamma_{a}$. The longitudinal dependence of the detuning is determined by the temperature or the strain along the fiber. $\Delta(z)=2 \pi\left[\nu_{p}-\nu_{s}-\delta \nu^{\operatorname{Res}}(z)\right] / \gamma_{a}$, where $\nu_{p}$ and $\nu_{s}$ are the pump and the Stokes frequencies and $\delta \nu^{\operatorname{Res}}(z)$ is the value of the pump-Stokes frequency shift for a resonant interaction at a given point. The boundary conditions correspond to the injection of a cw pump wave at $z=0$, i.e., $E_{p}(0)=E_{\mathrm{cw}}$, and a Stokes pulse at $z=L, E_{s}(L)=E_{\text {pulse }}(t)$. The function $E_{\text {pulse }}(t)$ is the sum of a small constant that accounts for the finite extinction ratio of the EOM and a square pulse that we smooth with a low-pass filter to reproduce a rise time of $100 \mathrm{ps}$. The normalized values of the optical fields $E_{x}$ are related to the power $P_{x}$ by the relation $E_{x}=K / \gamma_{a} \sqrt{\left(P_{x} Z_{0} / 2 A_{\text {eff }} n\right)}$, where $K$ is a Brillouin coupling constant, ${ }^{5} Z_{0}$ is the vacuum impedance, $A_{\text {eff }}$ is the effective mode area, and $n$ is the effective mode index.

Let us start the discussion with a very simple observation, which will be significant throughout the following development: The pump-field depletion is proportional to the product of the Stokes and the acoustic fields [Eq. (1a)]. The first consequence of this observation for our system is that high spatial resolution can be obtained, regardless of the finite acoustic damping time. The decay time of a $12-\mathrm{GHz}$ acoustic wave is $4.5 \mathrm{~ns}$ (corresponding to a $35-\mathrm{MHz}$ gain width at a $1.3-\mu \mathrm{m}$ working wavelength), and one may think that the interaction continues over that 
time; in fact, pump depletion lasts only as long as a Stokes field is present. One can therefore use probe pulses shorter than $4.5 \mathrm{~ns}$ to effectively obtain spatial resolution shorter than $47 \mathrm{~cm}$, as was demonstrated experimentally in the study reported in Ref. 3.

In our simulations we used parameters corresponding to the conditions of the experiment described in Ref. 3. Figure 1 shows the variations of the Brillouin loss width at half-maximum as a function of the probe pulse duration and for several values of the extinction ratio of the EOM. The curve obtained for a $30-\mathrm{dB}$ extinction ratio best matches the experimental result reported in Ref. 3. The linewidth exhibits a maximum of $120 \mathrm{MHz}$ for a pulse duration of $9 \mathrm{~ns}$. The width decreases quickly for shorter pulses, with an asymptotic value of $35 \mathrm{MHz}$. Typical Brillouin loss profiles obtained with 3-, 9-, and 50-ns pulse durations are shown in Fig. 2. In this figure and in the following, a null frequency shift corresponds to a stress-free fiber at ambient temperature. For the 9-ns pulses, a Gaussian-like profile is observed, as was reported for the experiment in Ref. 3. In Ref. 3, the authors attributed this behavior to interactions of individual phonons, and their discussion involved the notion of phonon coherence length and the distance that a phonon would travel in the fiber within the duration of the pulse. Let us reiterate that the mathematical model that we use here is classical and that the convection term of the acoustic wave has been neglected. If the experimental results from Ref. 3 can be reproduced in this framework, an alternative explanation has to be formulated.

The behavior of the system with pulses longer than 9 ns can be interpreted within a standard Fourier analysis. A 35- $\mathrm{MHz}$ broad resonance is excited with pulses the spectra of which are inversely proportional to their duration. The apparent gain is a convolution of the two spectra, which takes the features of the widest of the two spectra. For long probe pulses $(>50 \mathrm{~ns})$, the recorded spectra simply correspond to the Lorentzian profile of the Brillouin gain. For shorter pulses, the apparent gain broadens and takes a Gaussian-like profile that is attributed to the pulse spectrum.

One would expect this behavior to be maintained for very short pulses; however, as can be seen from Fig. 1, this is the case only when the switching device has a very high (infinite) extinction ratio. For example, when the pulses are generated by application of pulses of high-frequency modulation on an EOM, as reported in Ref. 6, the extinction ratio of the sidebands that are created can be very high. When one is using an EOM as in Ref. 3, the extinction ratio never normally exceeds $30 \mathrm{~dB}$, and in those conditions another effect prevails. This effect is illustrated in Fig. 3, which shows the temporal variations in the acoustic intensity as a 3 -ns pulse reaches a point of the fiber for 20-, 30-, and $40-\mathrm{dB}$ extinction ratios. The frequency shift is tuned to resonance at that point. Figure 3 shows that the background acoustic intensity from which the system starts has a strong influence on the maximum level reached within the $3-n s$ duration of the pulse (even though the three curves would reach the same asymptotic value in the case of very long pulses). The $\mathrm{cw}$
Stokes field leaking through the EOM interferes with the pump in every point of the sensing fiber and generates an acoustic wave. The intensity of this acoustic wave as a function of the frequency shift shows

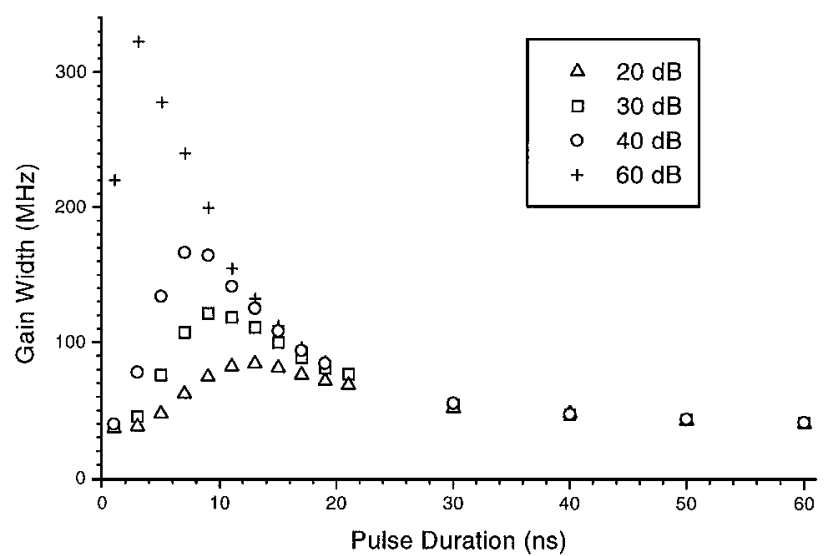

Fig. 1. Brillouin loss width as a function of pulse duration. $P_{p}=30 \mathrm{~mW}, P_{s}=2 \mathrm{~mW}$. The section of fiber that is considered is under strain $(200-\mathrm{MHz}$ shift compared with stress-free fiber) and is located in the middle of the sensor. Its length corresponds to the pulse duration (i.e., $10 \mathrm{~cm} / \mathrm{ns}$ ). The total sensing length is always at least three times longer than this section.

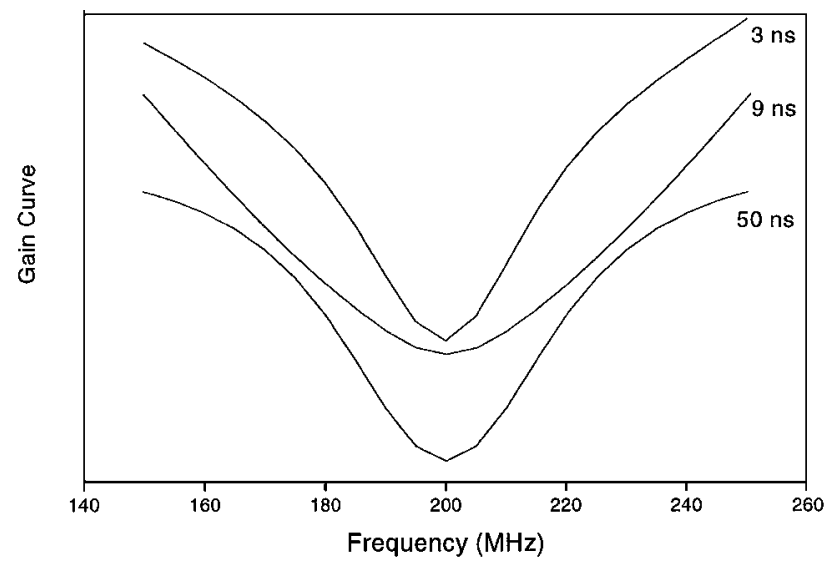

Fig. 2. Brillouin loss profiles. Extinction ratio, $30 \mathrm{~dB}$; $P_{p}=30 \mathrm{~mW}, P_{s}=2 \mathrm{~mW}$.

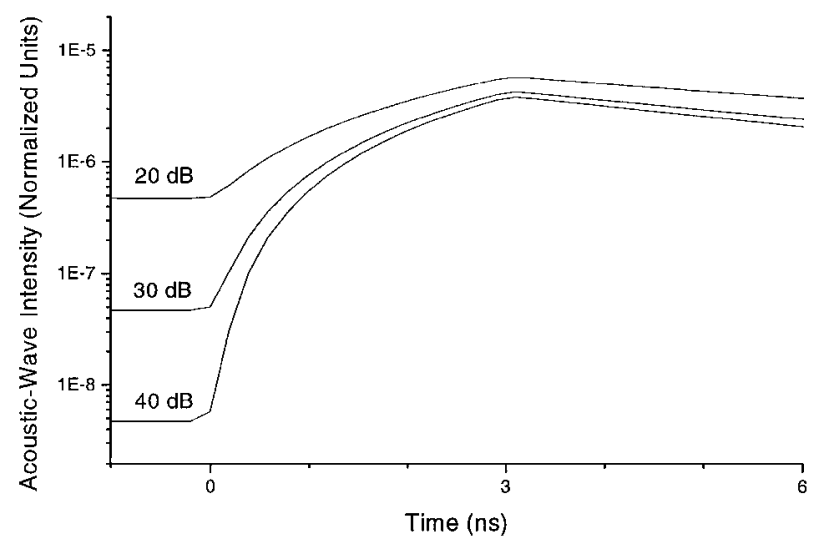

Fig. 3. Influence of the extinction ratio value on the local acoustic-wave intensity variations as a resonant probe pulse of 3 -ns passes. $P_{p}=30 \mathrm{~mW}, P_{s}=2 \mathrm{~mW}$. A point of a $30-\mathrm{cm}$ fiber section under strain $(200-\mathrm{MHz}$ shift) is considered. 


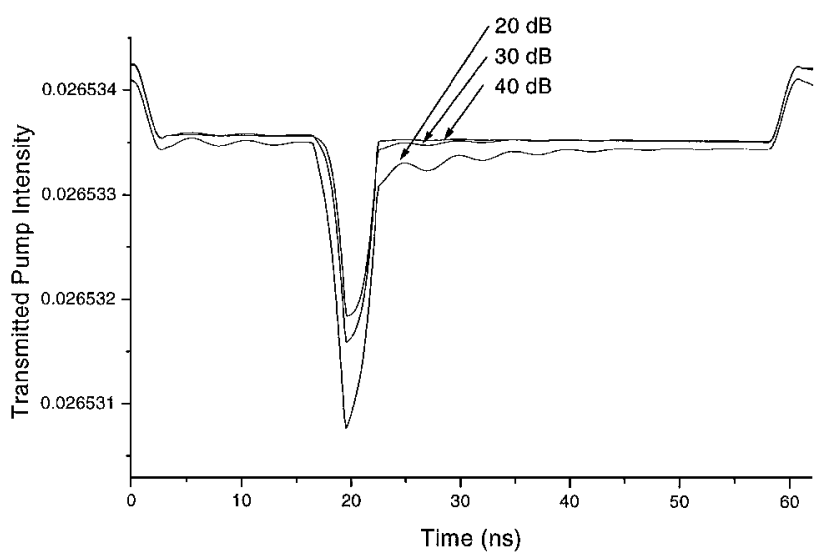

Fig. 4. Simulated traces of the transmitted pump for various values of the extinction ratio. A section of $30 \mathrm{~cm}$ obtained from a sensing length is under strain $(200-\mathrm{MHz}$ shift). The frequency shift is set for a resonant interaction in that section. $P_{p}=30 \mathrm{~mW}, P_{s}=2 \mathrm{~mW}$.

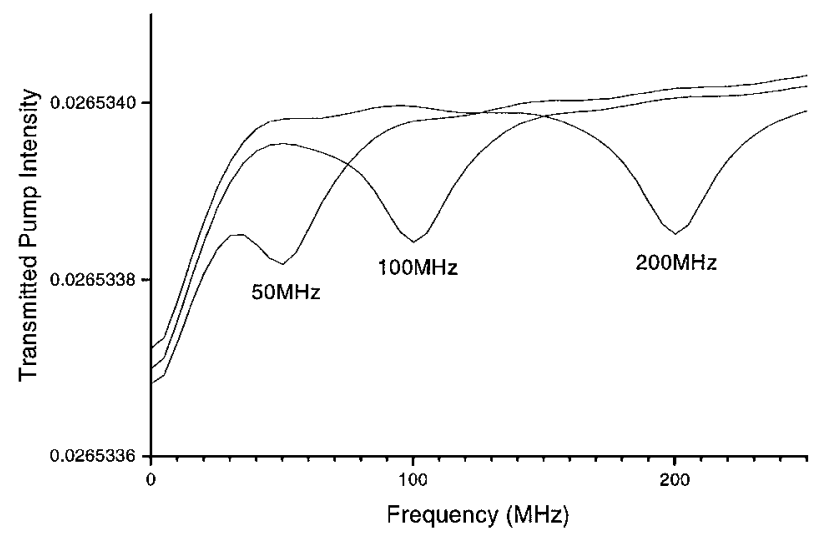

Fig. 5. Loss spectra obtained with 1-ns pulses, corresponding to a section of $10 \mathrm{~cm}$ of fiber under various strain levels (50-, 100-, and 200-MHz shifts). The sensing length is $3 \mathrm{~m}$. Extinction ratio; $30 \mathrm{~dB} ; P_{p}=30 \mathrm{~mW}, P_{s}=2 \mathrm{~mW}$.

a Lorentzian profile. In the case of a modest extinction ratio, the acoustic-wave growth associated with the passage of a short pulse is relatively small, and the resulting spectrum keeps the Lorentzian profile of the steady acoustic wave triggering the interaction. Pump depletion takes place efficiently through the sudden increase of the $E_{a} E_{s}$ term in Eq. (1a). For large extinction ratios the driving force associated with the pulse passage is the main source of the acoustic wave, which therefore takes a Gaussian-like profile associated with the short pulse spectrum. Note that, starting from a low level, the acoustic-wave intensity does not grow as much, and the resulting signal is smaller. Eventually, for a very high extinction ratio, one should consider the influence of the acoustic background associated with thermal agitation, which could be a source of noise in the recorded spectra.

Figure 4 shows the temporal variation of the transmitted pump for a sensing length of $3 \mathrm{~m}$ with a $30-\mathrm{cm}$ section fiber under strain $(200-\mathrm{MHz}$ shift), which would be scanned with a 3-ns pulse. The figure shows that a sufficient level of extinction ratio is re- quired if relaxation oscillations after the pulse passage are to be avoided. Clearly, the acoustic wave generated by the pulse continues to modulate the pump field when a cw Stokes field is present. Figure 5 shows Brillouin loss spectra obtained for a 30-dB extinction ratio, a $10-\mathrm{cm}$ section of a $3-\mathrm{m}$ fiber under various levels of strain (corresponding to 50-, 100-, and $200-\mathrm{MHz}$ shifts), and 1-ns probe pulses. The signal modulation is attributed to the relaxation oscillations. The global pump decrease for a null frequency shift corresponding to the average fiber condition is due to the cw Stokes leak. This result shows that one should use correction routines to be able to use those traces, especially when one is trying to measure the local fiber conditions close to the average condition in the whole fiber.

In conclusion, the sudden decrease of the spectral width that was observed in the experiments described in Ref. 3 results from the combination of a drop of the gain for very short pulses and the presence of an acoustic wave before the pulse passage. The intensity, and therefore the influence, of this steady acoustic wave is inversely proportional to the extinction ratio of the switching device used to generate the pulses. For a given extinction ratio, the Brillouin loss curve first broadens as the pulse duration is reduced. But since the gain produced by short pulses decreases, the acoustic wave created by the pulses can be of the same order of magnitude as the steady acoustic wave that is present before the pulse's arrival. Eventually, the recorded Brillouin spectrum reproduces the same features as the steady-state interaction, i.e., a Lorentzian profile of $35 \mathrm{MHz}$. Using low extinction ratios gives the advantages of a narrow linewidth and relatively large signals at high spatial resolution; however, it has the drawback of a distorted Brillouin gain curve. One can derive correction routines to be able to use the recorded signals, especially when the strain or temperature level that one wants to measure is close to the average fiber condition. This would probably require an iterative method in two steps: an estimate of the fiber condition based on the recorded spectra, followed by integration of Eqs. (1) over the sensing length, which would allow corrections of the recorded spectra and a more-precise estimate of the fiber condition.

V. Lecoeuche’s e-mail address is vl@ukc.ac.uk.

\section{References}

1. A. Fellay, L. Thevenaz, M. Facchini, M. Nikles, and P. Robert, in Optical Fiber Sensors, Vol. 16 of 1997 OSA Technical Digest Series (Optical Society of America, Washington, D.C., 1997), p. 324.

2. T. Horiguchi, K. Shimizu, T. Kurashima, and Y. Koyamada, Proc. SPIE 2507, 126 (1995).

3. X. Bao, A. Brown, M. DeMerchant, and J. Smith, Opt. Lett. 24, 510 (1999).

4. C. C. Chow and A. Bers, Phys. Rev. A 47, 5144 (1993).

5. V. Lecoeuche, S. Randoux, B. Segard, and J. Zemmouri, Phys. Rev. A 53, 2822 (1996).

6. M. Nikles, L. Thevenaz, and P. Robert, Opt. Lett. 21, 758 (1996). 